\title{
Primary Malignant Melanoma of the Esophagus
}

Dimitrios Karapiperis ${ }^{1}$, Spyridon Vrakas $^{2}$, Simone Ignatova ${ }^{3}$, George Tribonias ${ }^{4}$, and Thomas Franzen ${ }^{1}$

${ }^{1}$ Vrinnevi Hospital in Norrkoping

${ }^{2}$ Peripheral General Hospital of Peiraias Tzaneio

${ }^{3}$ Linköping University Hospital

${ }^{4}$ General Hospital of Nikaia Peiraia Agios Panteleimon

February 4, 2022

\begin{abstract}
We report a case of primary malignant melanoma of the esophagus.

Primary Malignant Melanoma of the Esophagus

Dimitrios Karapiperis ${ }^{1}$, Spyridon Vrakas $^{2}$, Simone Ignatova ${ }^{1}$, George Tribonias ${ }^{3}$, Thomas Franzen $^{1}$

${ }^{1}$ Department of Gastroenterology, Vrinnevi General Hospital of Norrkoping, Norrkoping, Sweden

${ }^{2}$ Department of Gastroenterology, Tzaneio General Hospital, Athens, Greece

${ }^{3}$ Department of Gastroenterology, General Hospital of Nikaia-Piraeus "Agios Panteleimon", Athens, Greece

\section{Correspondence}

Dimitrios Karapiperis, Vrinnevi General Hospital of Norrkoping, Gamla Ovagen 25, 60379 Norrkoping, Sweden Email: dr.karapiperis@gmail.com

\section{Key Clinical Message}

Primary malignant melanoma of the esophagus (PMME) is a rare primary neoplasm of the esophagus. Although it is rare it must be borne in mind, as early and correct diagnosis could offer the patient a better prognosis.
\end{abstract}

\section{Abstract}

We report a case of primary malignant melanoma of the esophagus.

\section{Keywords}

Melanoma, Esophagus

A 70 year old female was admitted to our hospital with a history of dysphagia for solids and weight loss. An endoscopy was performed and revealed an ulcerated mass involving the lower third of esophagus (Figure 1). Histology revealed a diffuse proliferation of atypical epithelioid amelanotic cells with irregular nuclear borders and sometimes prominent nucleoli associated with tumor necrosis. Immunohistochemical findings included positive Melan-A, SOX-10, HMB-45, focally positive S-100 and negative CK20, CK7, Synaptophysin, TTF-1, LCA and CD-117 (Figure 2). Positron emission tomography-CT (PET-CT) showed an increased uptake of 
fluorodeoxy-glucose in the lower part of esophagus (Figure 3). After a thorough search for a primary tumor from skin, ocular and mucosal sites a diagnosis of primary malignant melanoma of esophagus (PMME) was made. A subtotal esophagectomy was done, the patient had a good clinical outcome and was discharged 10 days after surgery.

Primary malignant melanoma of the esophagus (PMME) accounts for $0.1-0.2 \%$ of all primary neoplasms of the esophagus ${ }^{1}$. Most commonly, it affects patients between the sixth and seventh decade of life and there is a male predominance ${ }^{2}$. Most common symptoms are dysphagia, chest pain, and weight loss. PMME is usually fatal with a mean survival time 13.4 months $^{3}$.

\section{CONSENT STATEMENT}

Informed consent has been obtained from patient for the publication of this clinical image.

\section{AUTHOR CONTRIBUTIONS}

DK, SV, SI, GT and TF: contributed to the writing and approval of the final manuscript.

\section{CONFLICT OF INTEREST}

None declared.

\section{ETHICAL STATEMENT}

Written informed consent was obtained from patient. This case report did not receive any funding. Authors have access to all source data for this case report.

\section{DATA AVAILABILITY STATEMENT}

Data supporting the findings of this study are available from the corresponding author on request.

1) Cheung MC, Perez EA, Molina MA, Jin X, Gutierrez JC, Franceschi D, Livingstone AS, Koniaris LG. Defining the role of surgery for primary gastrointestinal tract melanoma. J Gastrointest Surg. 2008;12(4):731-8.

2) Fukuda S, Ito H, Ohba R, Sato Y, Ohyauchi M, Igarashi T, Obana N, Iijima K. A Retrospective study, an initial lesion of primary malignant melanoma of the esophagus revealed by endoscopy. Intern Med. 2017;56(16):2133-7.

3) Sabanathan S, Eng J, Pradhan GN. Primary malignant melanoma of the esophagus. Am J Gastroenterol. 1989;84:1475-1481. 

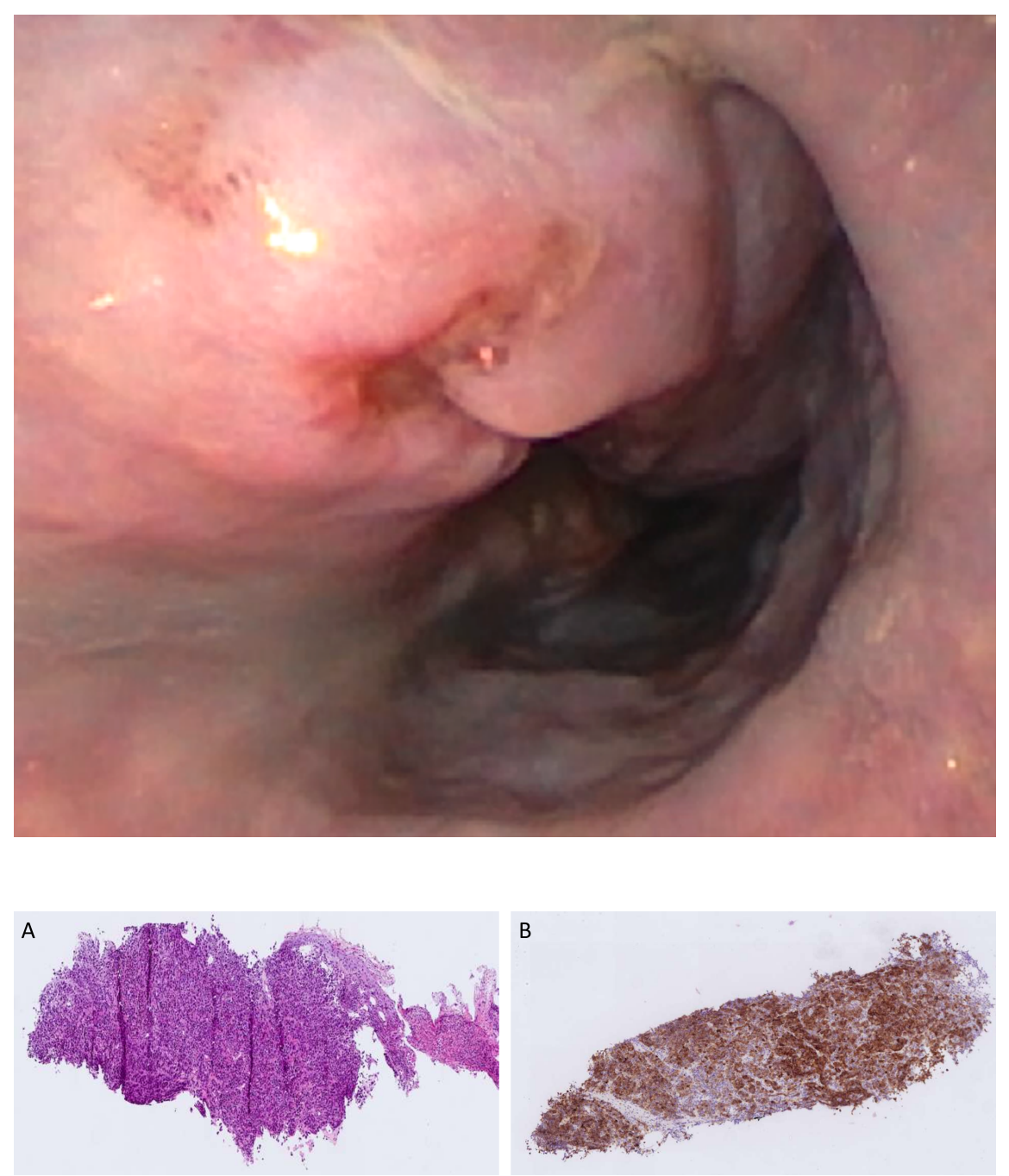

B



c


E

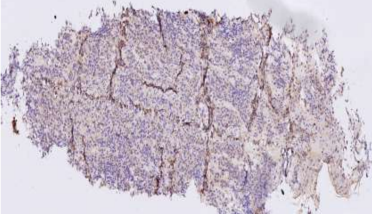






